BULL. AUSTRAL. MATH. SOC.

$16 A 72,16 A 32,17 A 01$

VOL. 29 (1984), 215-229.

\title{
HNN-EXTENSIONS OF ALGEBRAS \\ AND APPLICATIONS
}

\author{
Hans-Christian Mez
}

\begin{abstract}
The classic HNN-embedding theorem for groups does not transfer to associative rings or algebras. In its first part this paper presents constructions which provide such a theorem if an additional condition is put on the isomorphic subalgebras or if one restricts to algebras over fields and drops the associativity. The main part of the paper deals with applications of these results. For example, it is known that every existentially closed group is $\omega$-homogeneous. It is shown that the corresponding is false for existentially closed associative $\Delta$-algebras but true for existentially universal nonassociative $K$-algebras. Furthermore, orthogonal sequences of idempotents in existentially closed associative $\Delta$-algebras over a regular ring $\Delta$ are investigated. It is shown that the conjugacy class of such a sequence depends only on a corresponding order sequence. In particular, in every existentially closed $K$-algebra all idempotents different from 0 and $I$ are conjugated.
\end{abstract}

\section{Introduction}

A fundamental instrument in combinatorial group theory is a theorem of Higman, Neumann and Neumann [9]. It says that every isomorphism between subgroups of a group can be extended to an inner automorphism of a

Received 17 October 1983.

Copyright Clearance Centre, Inc. Serial-fee code: 0004-9727/84 $\$ \mathrm{~A} 2.00+0.00$. 
supergroup. The corresponding theorem for skew fields was shown by Cohn [2]. In a recent paper Dicks [3] investigates HNN-constructions for associative rings.

The rings and algebras we consider contain a multiplicative identity 1 and are not necessarily commutative. It is continually indicated whether rings are associative or nonassociative (that is, not necessarily associative). Always, the identity is inherited by subrings, preserved by ring homomorphisms and acts unitally on all modules. In connection with algebras, $\Delta$ denotes a fixed commutative associative ring and $K$ a fixed field.

For a given associative $\Delta$-algebra $B$ and an algebra isomorphism $\psi: C \rightarrow D$ between subalgebras we can always form the associated (associative) HNN-construction

$$
A:=\left\langle B, t, s ; t s=s t=1, t e t^{-1}=\psi(c) \text { for all } c \in c\right\rangle,
$$

see [3], but in contrast to groups or division rings, the natural homomorphism $n: B \rightarrow A$ defined by $n(b):=b$ needs not to be injective. In order to apply this construction to problems in combinatorial or modeltheoretic ring theory, the injectivity is very desirable.

Section 1 presents HNN-embedding theorems if we assume that the isomorphic subalgebras $C, D$ are (von Neumann) regular or if we restrict to $K$-algebras and drop the associativity. Both cases are handled simultaneously. The main tools are well-known things like free products with amalgamations, which exist according to papers of Cohn [1] and Dididze [4], [5], shift automorphisms and skew polynomial algebras. The first case, concerning regular subalgebras of associative $\Delta$-algebras, now follows as well from work done by Dicks [3], that is, from results based on his Theorem 9. Concerning nonassociative $K$-algebras, the outcome is a general HNN-theorem as for groups. Additionally, in the HNN-extension, we get that each of the elements $t, t^{-1}$ associates with all pairs of the extension. Just this fact makes the theorem applicable to various problems.

The next and final section applies the HNN-extensions of algebras in several situations. The HNN-theorem for groups implies that existentially closed groups are $\omega$-homogeneous and therefore uniquely determined up to 
partial isomorphism by the isomorphism types of their finitely generated subgroups. After clarifying the terminology, we discuss the corresponding question for algebras. It is shown that there are arbitrarily large existentially closed, even existentially universal associative $\Delta$-algebras which are not $\omega$-homogeneous. On the other hand, existentially universal nonassociative $K$-algebras are found to be $\omega$-homogeneous.

Next, we concentrate on associative $\Delta$-algebras. Now HNN-extensions are only available if regular subalgebras are considered. But in case $\Delta$ is regular, including $\Delta=K$, idempotents and even orthogonal sequences of idempotents generate regular subalgebras. We show that existentially closed associative $\Delta$-algebras contain a lot of such elements and sequences and prove theorems about them. If $\Delta$ is regular, it turns out that in algebraically closed associative $\Delta$-algebras two orthogonal sequences of idempotents $a_{1}, \ldots, a_{n}$ and $b_{1}, \ldots, b_{n}$ are conjugated if and only if $o\left(a_{i}\right)=O\left(b_{i}\right)$ for $i=1, \ldots, n$ and

$$
o\left(1-\left(a_{1}+\ldots+a_{n}\right)\right)=O\left(1-\left(b_{1}+\ldots+b_{n}\right)\right)
$$

where $O(a)$ denotes the order with respect to the $\Delta$-module structure. Hence, in case $\Delta=K$, all orthogonal sequences of $n$ non-zero idempotents split into two conjugacy classes. The distinguishing property is whether the sum equals 1 or not. For $n=1$ this means that all idempotents different from 0 and 1 are conjugated.

Finally, we briefly discuss the situation if we do not require a multiplicative identity in our algebras.

The HNN-extension theorems and some of the applications are based on parts of the author's dissertation completed in 1982 at the University of Freiburg.

\section{HNN-extensions of algebras}

First, we study an example for associative $\Delta$-algebras which is based on an idea of Cohn [2, Example 1, p. 95]. Throughout the paper $[X]_{\Delta}$ denotes the $\Delta$-submodule, $(X)_{\Delta}$ the $\Delta$-subalgebra and $(X)^{A}$ the ideal generated by a set $X$ in a $\Delta$-algebra $A \cdot \Delta(X)$ denotes the free noncommutative associative $\Delta$-algebra over $X$. 
EXAMPLE 1. Let $J$ be the ideal generated by $\{v x-1, y z\}$ in $\Delta(v, x, y, z\rangle$, and let $A_{\Delta}:=\Delta\langle v, x, y, z\rangle / J$. The polynomial algebras $\Delta(x), \Delta\langle y\rangle$ are $\Delta$-subalgebras of the associative $\Delta$-algebra $A_{\Delta}$, and let $\psi_{\Delta}: \Delta\langle X\rangle \rightarrow \Delta\langle y\rangle$ be the $\Delta$-algebra isomorphism defined by $\psi_{\Delta}(x):=y$.

In view of a discussion concerning whomogenity in Section 2, we prove the following:

REMARK 2. There are no associative $\Delta$-algebras $S, T$ with $\Delta$-algebra isomorphism $\phi: S \rightarrow T$ such that $S \cap T \supseteq A_{\triangle}$ and $\phi$ extends $\psi_{\Delta}$

Proof. Assume, if possible, that such $S, T$ and $\phi: S \rightarrow T$ exist. Then

$z=I \cdot z=\phi(1) \cdot z=\phi(\nu \cdot x) \cdot z$

$$
=\phi(v) \cdot \phi(x) \cdot z=\phi(v) \cdot y \cdot z=\phi(v) \cdot 0=0,
$$

but we know $z \neq 0$ in $A_{\Delta}$, a contradiction.

COROLLARY 3. There is no associative $\Delta$-algebra $S \supseteq A_{\Delta}$ with $\Delta$-algebra automorphism $\phi: S \rightarrow S$ extending $\psi_{\Delta}$.

This corollary definitely demonstrates that there is no global HNNextension theorem for associative $\Delta$-algebras. Now let us turn to the positive results announced in the introduction. The initial step is a theorem whose analogue for groups admits a rather simple proof (just a semidirect product with an infinite cyclic group inducing the automorphism).

THEOREM 4. Let $S$ be a not necessarily associative $\Delta$-algebra and $\phi: S \rightarrow S$ be a $\Delta$-algebra automorphism. Then there exists a $\Delta$-algebra $A$ extending $S$ with a viit $t \in A$ such that

(i) for every $m \in \mathbb{Z}$ the element $t^{m}$ associates with all pairs of $A$,

(ii) for alz $s \in S$ we have $\phi(s)=t s t^{-1}$.

If $S$ is associative then $A$ can be chosen associative as well. 
Proof. Obviously, we define $A$ as the skew Laurent polynomial algebra

$$
s\left\langle t, t^{-1} ; \phi\right\rangle:=\left\{\sum_{k=-n}^{n} s_{k} t^{k} \mid n \in N_{0}, s_{k} \in S\right\}
$$

whose multiplication is based on $t^{ \pm l} \cdot s:=\phi^{( \pm l)}(s) t^{ \pm 1}$. To verify that $A$ is a not necessarily associative $\Delta$-algebra extending $S$, we need only that $\phi$ is a $\Delta$-module automorphism which fixes the identity 1 . Using that $\phi$ is a $\Delta$-algebra automorphism, we get that $t^{m}(m \in \mathbb{Z})$ associates with all pairs from $A$ and that $A$ is associative if $S$ is. Finally, $t s t^{-1}=(t s) t^{-1}=(\phi(s) t) t^{-1}=\phi(s)$ for all $s \in S$.

DEFINITION 5. An associative ring $R$ is called (von Neumann) regular if for every $x \in R$ there is a $y \in R$ such that $x y x=x$.

A comprehensive survey of regular rings offers the book of Goodearl [8]. Next we formulate the two HNN-extension theorems and prove them simultaneously. The first, concerning associative $\Delta$-algebras, now follows as well from Dicks [3], that is, from his Theorem 9.

THEOREM 6. Let $B$ be an associative $\Delta$-algebra and $\psi: C \rightarrow D$ be an algebra isomorphism between (von Neumann) regular subalgebras $C, D$. Then there exists an associative $\Delta$-algebra $A$ extending $B$ with a vonit $t \in A$ such that $\phi(c)=t_{c t}^{-1}$ for all $c \in C$.

THEOREM 7. Let $B$ be a not necessarily associative K-algebra and $\psi: C \rightarrow D$ be an algebra isomorphism between subalgebras $C, D$. Then there exists a not necessarily associative $K$-algebra $A$ extending $B$ with a voit $t \in A$ such that

(i) for every $m \in \mathbb{Z}$ the element $t^{m}$ associates with all pairs of $A$,

(ii) $\psi(c)=\operatorname{tet}^{-1}$ for all $c \in C$.

Proof of both theorems. We use the following two results.

1 (Cohn [1, Theorem 4.7, p. 393. If $\Lambda$ is a $\Delta$-algebra, then every $\Lambda$-ring as well"]). The (associative) free product of a family of associative $\Delta$-algebras with one amalgamated regular subalgebra always 
exists.

2 (Dididze [4], [5]). The free product of a family of nonassociative $K$-algebras with one amalgamated subalgebra always exists. For a short sketch, without identity, see MR20\#6448. With identity 1 , just let there $Z$ contain $I$ considerea as the empty word.

Now let $\left(B_{i}, C_{i}, D_{i}, \Psi_{i}\right)$ for $i \in \mathbb{Z}$ be disjoint copies of the given situation $(B, C, D, \psi)$ which is identified with $\left(B_{0}, C_{0}, D_{0}, \psi_{0}\right)$.

Let

$$
\begin{aligned}
S_{0} & :=B_{0}, \\
S_{2 m+1} & :=S_{2 m_{D_{m}}=C_{m+1}{ }_{m+1},} \\
S_{2 m+2} & :=B_{-(m+1)_{D_{-}(m+1)}}=C_{-m} S_{2 m+1} .
\end{aligned}
$$

Forming the union of the ascending chain $\left\{s_{n} \mid n \in \mathrm{N}_{0}\right\}$, we get an algebra extension $S$ of $B$. Let $\phi: S+S$ be the left-right shift automorphism (each factor belonging to $B_{i}$ is transported to $B_{i+1}$ ). We have $\phi(c)=\phi\left(c_{0}\right)=c_{1}=\psi_{0}\left(c_{0}\right)=\psi(c)$ for all $c \in C \cdot$ Finally, an application of Theorem 4 terminates the proof.

\section{Applications}

The HNN-theorem for groups implies that every existentially closed group is $w$-homogeneous. Let us discuss the corresponding for algebras.

Algebraically closed, existentially closed and existentially universal structures are currently investigated for many classes. Using existential formulae, these structures are defined model-theoretically in Hirschfeld and Wheeler [10] and in Eklof [6]. Note that existentially universal is stronger than existentially closed which implies algebraically closed. For concrete algebraic classes - as groups, algebras or division rings - they can easily be described algebraically by the solvability of certain sets of equations and inequations. For associative $\Delta$-algebras such a description can be found in [7]. Here we use in (in-)equations the symbols,$+ \cdot$, $-(1,0,1$, and $\lambda \cdot()$ for every $\lambda \in \Delta$ and discuss the classes: 
(I) associative $\Delta$-algebras;

(II) nonassociative, that is, not necessarily associative, $\Delta$-algebras.

In both classes every algebra $A$ can be embedded in an existentially closed algebra $B$, even in an existentially universal algebra $C$. Additionally we can have

$$
|B| \leq \max \left\{|A|,|\Delta|, \kappa_{0}\right\}
$$

and

$$
|C| \leq \max \left\{|A|, 2^{\max \left\{|\Delta|, \aleph_{0}\right\}}\right\}
$$

Consequently, theorems about algebraically closed, existentially closed or existentially universal algebras provide embedding theorems for the individual classes with cardinality bounds.

DEFINITION 8 . Let $A$ be a not necessarily associative $\Delta$-algebra. $A$ is called whomogeneous if for every algebra isomorphism $\psi: C \rightarrow D$ between finitely generated subalgebras of $A$ and every $a \in A$ there is an isomorphism $\hat{\psi}:(C, a)_{\Delta} \rightarrow E \subseteq A$ extending $\psi$.

PROPOSITION 9. The associative $\Delta$-algebra $A_{\Delta}$ defined in Example I cannot be embedded in any whomogeneous associative $\Delta$-algebra. Consequently, there are arbitrarily large existentially closed, even existentially universal associative $\Delta$-algebras which are not w-homogeneous.

Proof. Assume, if possible, that there is a $\omega$-homogeneous associative $\Delta$-algebra $B$ extending $A_{\Delta}=\langle v, x, y, z)_{\Delta}$. Let $\psi_{\Delta}:\langle x\rangle_{\Delta}+\langle y\rangle_{\Delta}$ be the map defined in Example 1. Applying the $\omega-$ homogenity six times (back and forth), we get two $\Delta$-algebras $S, T \subseteq B$ and a $\Delta$-algebra isomorphism $\phi: S \rightarrow T$ such that $S \cap T \supseteq A_{\Delta}$ and $\phi$ extends $\Psi_{\Delta}$. Because of Remark 2 this is impossible.

Next we realize that nonassociative $K$-algebras behave differently. Since we have the unrestricted HNN-theorem available, our first guess is that it is possible to proceed as with groups. But during the argument we need to express that a new element associates with all nonassociative semi- 
group words over finitely many given generators. This cannot be formulated within a single existential sentence. However, we are still able to show the $\omega$-homogenity of $a l l$ existentially universal nonassociative $K$-algebras which form a cofinal subclass of the existentially closed ones.

THEOREM 10. Every existentially universal nonassociative $K$-algebra is whomogeneous.

Proof. Let $B$ be an existentially universal nonassociative $K$-algebra, $\psi:\left\langle c_{1}, \ldots, c_{n_{l}}\right\rangle_{K} \rightarrow\left\langle d_{1}=\psi\left(c_{1}\right), \ldots, d_{n}=\psi\left(c_{n}\right)\right\rangle_{K}$ an isomorphism and $b \in B$. We have to extend $\psi$ to an isomorphism $\tilde{\psi}:\left\langle b, c_{1}, \ldots, c_{n}\right\rangle_{K} \rightarrow E \subseteq B$.

For variables $x, y$ we define

$\Theta(x, y, b, \vec{c}, \vec{d})$ $:=\left\{d_{i}=\left(x c_{i}\right) y \mid i=1, \ldots, n\right\} \cup\{x y=y x=1\} \cup\{$ "every permutation of $x, z, z^{\prime}$ and of $y, z, z^{\prime}$ associates" $\left.\mid z, z^{\prime} \in\left\langle x, y, b, c_{1}, \ldots, c_{n}\right\rangle_{K}\right\}$.

Now choose the extension $A$ of $B$ according to Theorem 7 .

Consequently, in $A$ the statements $\theta\left(t, t^{-1}, b, \vec{c}, \vec{d}\right)$ are true. Since $B$ is existentially universal and $\theta$ has the required form (see Hirschfeld and Wheeler [10, Chapter 1, \$2]), we may conclude that there are $u_{s} v \in B$ such that $\Theta(u, v, b, \vec{c}, \vec{d})$ hold. Of course $v=u^{-1}$.

Define $\tilde{\psi}:\left\langle b, c_{1}, \ldots, c_{n}\right\rangle_{K} \rightarrow B$ by $\tilde{\psi}(g):=(u g) v$ which is obviously a $K$-linear map. Next, let us check that $\tilde{\psi}$ preserves multiplications. For $g, h \in\left\langle b, c_{1}, \ldots, c_{n}\right\rangle_{K}$ we have $\tilde{\psi}(g) \tilde{\psi}(h)=((u g) v)((u h) v)=((u g) v)(u(h v))=(u g)(v(u(h v)))$
$=(u g)((v u)(h v))=(u g)(h v)=((u g) h) v=(u(g h)) v=\tilde{\psi}(g h)$. To show injectivity, suppose $0=\tilde{\psi}(g)=(u g) v$. Then

$$
0=v(((u g) v) u)=v((u g)(v u))=v(u g)=(v u) g=g .
$$

Since $\tilde{\psi}\left(c_{i}\right)=\left(u c_{i}\right) v=d_{i}=\psi\left(c_{i}\right)$, we know that $\tilde{\psi}$ extends $\psi$, and we are done.

COROLLARY 11. Two existentially zoniversal nonassociative $K$-algebras are partially isomorphic, that is, $L_{\text {oow }}$-elementarily equivalent, if and 
only if both have the same isomorphism types of finitely generated subalgebras.

PROBLEM 12. For countably generated existentially zoniversal nonassociative K-algebras "partially isomorphic" reduses to "isomorphic" in Corollary 1l. But do such algebras exist? For example, there are comtable existentially universal fields (countable universal domains).

Next, let us discuss associative $\Delta$-algebras. In a joint paper with Eklof [7] the algebraically closed, existentially closed and existentially universal members of this class are investigated. First without underlying multiplicative identity, which permits more extension constructions, for example, free products and direct sums. One of the main tools is realizing $\Delta$-linear mappings of algebras by left-right multiplications. Some of the results (see [7]) remain valid if we insist, as we do in this note, on an identity 1 inherited by subalgebras and preserved by algebra homomorphisms. For example, every algebraically closed associative $K$-algebra is existentially closed and simple, even for all elements $a, b$ with $a \neq 0$ the equation $b=u a v$ is solvable. The known proofs for the fact that every nontrivial algebraically closed group is existentially closed and simple require the HNN-theorem.

In order to employ the HNN-theorem for associative $\Delta$-algebras, we need regular subalgebras. If $\Delta$ is assumed to be regular, for example, $\Delta=K$, we can get regular subalgebras via idempotents.

DEFINITION 13 (see Jacobson [11, Chapter 3,57]). Let $A$ be a $\Delta$-algebra. An $a \in A$ is called idempotent if $a^{2}=a$. A sequence $a_{1}, \ldots, a_{n} \in A$ consisting of idempotents is called orthogonal if $a_{i} a_{j}=0$ for all $i \neq j$. (By definition, a single idempotent forms an orthogonal sequence.)

First, we show that existentially closed associative $\Delta$-algebras contain a lot of idempotents and orthogonal sequences. Then, specializing to regular $\Delta$, we prove theorems about them.

THEOREM 14. Let $A$ be an existentially closed associative $\Delta$-algebra. For every $n \geq 1$ there are infinitely many orthogonal sequences of $n$ idempotents in $A$ such that no two of these sequences have a common element. 
Proof. Let $B$ be the (unrestricted) direct product of infinitely many copies of $A$. Identify $A$ with the diagonal of $B$, and $B$ can be viewed as an algebra extension of $A$.

The infinite sequence

$$
(1,0,0, \ldots),(0,1,0, \ldots),(0,0,1,0, \ldots), \ldots
$$

consists of idempotents contained in $B \backslash A$ and is orthogonal. For $n \geq 1$ take the statement

$$
\begin{aligned}
\exists x_{1} \ldots \exists x_{n}\left\{\left\{x_{i}^{2}=x_{i} \mid i=1, \ldots, n\right\}\right. & \\
& \left.\wedge \wedge\left\{x_{i} x_{j}=0 \mid i, j=1, \ldots, n ; i \neq j\right\}\right) .
\end{aligned}
$$

Since it is true in $B$ and since $A$ is existentially closed, it must be true in $A$ as well. Hence there is an orthogonal sequence $a_{1}, \ldots, a_{n}$ of idempotents in $A$. Now add in the above statement the condition $\wedge\left\{x_{i} \neq a_{j} \mid i, j=1, \ldots, n\right\}$, and we get a new sequence, and so on.

Using the technique in the proof of Theorem 14, we could get more sets of orthogonal sequences of $n$ idempotents in $A$ with specific properties, for example, $a_{1}+\ldots+a_{n}=1$ or $a_{1}+\ldots+a_{n} \neq 1$.

LEMMA 15. Let $\Delta$ be regular and $A$ be an associative $\Delta$-algebra. Then every orthogonal sequence of idempotents of $A$ generates a regular cormutative subalgebra.

Proof. If $C$ is any $\Delta$-algebra, let $C \times) \Delta$ denote the direct sum as $\Delta$-modules with the multiplication rule $(c, \lambda)(d, \mu):=(c d+\lambda d+\mu c, \lambda \mu)$. This is the usual adjoining of a new identity (the pair $(0,1)$ ), and $C \times) \Delta$ is a $\Delta$-algebra containing $C$ as an ideal.

Let $a_{1}, \ldots, a_{n}$ be an orthogonal sequence of idempotents of $A$. Then $\left\langle a_{1}, \ldots, a_{n}\right\rangle_{\Delta}=\left[a_{1}, \ldots, a_{n}, 1\right]_{\Delta}$ which is a $\Delta$-algebra homomorphic image of $\left.\Delta^{n} x\right) \Delta$, which is commutative, where $\Delta^{n}$ is the direct product of $n$ copies of $\Delta$. According to Goodearl [8, Chapter 1], $\left.\Delta^{n} x\right) \Delta$ is regular and consequently $\left\langle a_{1}, \ldots, a_{n}\right\rangle_{\Delta}$ as well.

If $a$ is an element of some $\Delta$-module, let $O(a):=\{\lambda \in \Delta \mid \lambda a=0\}$, 
the so-called order ideal of $a$. Next, in case $\Delta$ is regular, we show that in every algebraically closed associative $\Delta$-algebra the conjugacy class of an orthogonal sequence of idempotents depends only on the order ideals of the individual idempotents and of the difference between their sum and the identity. For $n=1$ this describes the conjugacy class of an arbitrary idempotent.

THEOREM 16. Let $\Delta$ be regular, $A$ be an algebraically closed associative $\Delta$-algebra and $a_{1}, \ldots, a_{n}, b_{1}, \ldots, b_{n}$ be two orthogonal sequences of $n$ idempotents of $A$. Then there is a wit $u \in A$ such that $b_{i}=u a_{i} u^{-1}$ for $i=1, \ldots, n$ if and only if $o\left(a_{i}\right)=o\left(b_{i}\right)$ for $i=1, \ldots, n$ and $o\left(1-\left(a_{1}+\ldots+a_{n}\right)\right)=o\left(1-\left(b_{1}+\ldots+b_{n}\right)\right)$.

Proof. One direction is obvious since conjugations preserve order ideals. Let us turn to the interesting part of the proof. Suppose the two orthogonal sequences of idempotents satisfy $O\left(a_{i}\right)=O\left(b_{i}\right)$ for $i=1, \ldots, n$ and $o\left(1-\left(a_{1}+\ldots+a_{n}\right)\right)=o\left(1-\left(b_{1}+\ldots+b_{n}\right)\right)$. According to Lemma 15 , the subalgebras $\left\langle a_{1}, \ldots, a_{n}\right\rangle_{\Delta}=\left|1, a_{1}, \ldots, a_{n}\right|_{\Delta}$ and $\left\langle b_{1}, \ldots, b_{n}\right\rangle_{\Delta}=\left|1, b_{1}, \ldots, b_{n}\right|_{\Delta}$ are regular.

Define the map $\psi:\left\langle a_{1}, \ldots, a_{n}\right\rangle_{\Delta} \rightarrow\left\langle b_{1}, \ldots, b_{n}\right\rangle_{\Delta}$ by

$$
\psi\left(\lambda+\sum_{i=1}^{n} \lambda_{i} a_{i}\right):=\lambda+\sum_{i=1}^{n} \lambda_{i} b_{i} .
$$

Obviously, we know that $\psi$ is a $\Delta$-algebra isomorphis if we can verify that $\psi$ is well-defined and injective. This is done if we can show that

$$
\lambda+\sum_{i=1}^{n} \lambda_{i} a_{i}=0
$$

if and only if

$$
\lambda+\sum_{i=1}^{n} \lambda_{i} b_{i}=0
$$

for all $\lambda, \lambda_{1}, \ldots, \lambda_{n} \in \Delta$.

Suppose 


$$
0=\lambda+\sum_{i=1}^{n} \lambda_{i} a_{i}
$$

For arbitrary $j \in\{1, \ldots, n\}$, multipling by $a_{j}$ results in

$$
0=\lambda a_{j}+\sum_{i=1}^{n} \lambda_{i} a_{i} a_{j}=\left(\lambda+\lambda_{j}\right) a_{j},
$$

that is, $\lambda_{j} a_{j}=-\lambda a_{j}$. Because of $O\left(a_{j}\right)=O\left(b_{j}\right)$, we may conclude $\left(\lambda+\lambda_{j}\right) b_{j}=0$, that is, $\lambda_{j} b_{j}=-\lambda b_{j}$. Then we have

$$
0=\lambda+\sum_{i=1}^{n} \lambda_{i} a_{i}=\lambda-\sum_{i=1}^{n} \lambda a_{i}=\lambda\left(1-\left(a_{1}+\ldots+a_{n}\right)\right) .
$$

Since $o\left(1-\left(a_{1}+\ldots+a_{n}\right)\right)=O\left(1-\left(b_{1}+\ldots+b_{n}\right)\right)$, this implies

$$
0=\lambda\left(1-\left(b_{1}+\ldots+b_{n}\right)\right)=\lambda-\sum_{i=1}^{n} \lambda b_{i}=\lambda+\sum_{i=1}^{n} \lambda_{i} b_{i}
$$

which is the desired result. The converse follows from a symmetrical argument.

Next we apply Theorem 6 on $A$ together with

$$
\psi:\left\langle a_{1}, \ldots, a_{n}\right\rangle_{\Delta} \rightarrow\left\langle b_{1}, \ldots, b_{n}\right\rangle_{\Delta}
$$

and get an associative $\Delta$-algebra extension $H$ of $A$ and a unit $t \in H$ such that $\psi(e)=$ tet $^{-1}$ for all $e \in\left\langle a_{1}, \ldots, a_{n}\right\rangle_{\Delta}$, in particular, $b_{i}=t a_{i} t^{-1}$ for $i=1, \ldots, n$. Now $H$ satisfies the statement

$$
\exists x \exists y\left(x y=y x=1 \wedge \wedge\left\{b_{i}=x a_{i} y \mid i=1, \ldots, n\right\}\right) .
$$

Since $A$ is algebraically closed, it satisfies this statement as well, and the proof is completed.

COROLLARY 17. Let $A$ be an algebraically closed associative $K$-algebra and $a_{1}, \ldots, a_{n}, b_{1}, \ldots, b_{n}$ be two orthogonal sequences of non-zero idempotents of $A$. Then there is a wit $u \in A$ such that $b_{i}=u a_{i} u^{-1}$ for $i=1, \ldots, n$ if and only if either 


$$
a_{1}+\ldots+a_{n}=1=b_{1}+\ldots+b_{n}
$$

or

$$
a_{1}+\ldots+a_{n} \neq 1 \neq b_{1}+\ldots+b_{n} \text {. }
$$

In particular, the infinitely many idempotents of $A$ which are different from 0 and 1 form one conjugacy class.

The last corollary supplements the earlier mentioned properties of algebraically closed associative $K$-algebras. Since HNN-extensions always exist for nonassociative $K$-algebras, it is possible to analyse under which conditions two arbitrary sequences of $n$ elements of an algebraically closed nonassociative $K$-algebra are conjugated. For example, the analogue of Corollary 17 holds but, of course, more general results are true. But instead of doing this, let us finally discuss in brief what happens if we do not require in our algebras a multiplicative identity respected by subalgebras and by algebra homomorphisms.

Every $\Delta$-algebra $A$, associative or not, can be adjoined an identity via $A \times) \Delta$, as described in the proof of Lemma 15. For a given algebra $B$ with an isomorphism $\psi: C \rightarrow D$ between subalgebras, it still makes sense to talk about an HNN-extension $A$ if at least $A$ has an identity (necessary for units!). Using $B \times$ ) $\Delta$ and $\bar{\psi}: C \times) \Delta \rightarrow D x$ ) $\Delta$ defined by $\bar{\psi}((c, \lambda)):=(\psi(c), \lambda)$, we reduce the problem to results already proved. Thus, Theorems 4 and 7 still hold while Theorem 6 , at least if based on the described argument, needs that $\Delta$ is regular (then $C x$ ) $\Delta$, $D x) \Delta$ are regular as well). Note that Remark 2 and its corollary are still true; the algebras $S, T$ need not have an identity $\left(A_{\Delta}\right.$ has 1 by definition and $\phi(1)=\psi_{\Delta}(1)=1$ ).

If we consider algebras which do not necessarily contain an identity, the notion of algebraically closed, existentially closed and existentially universal algebras changes. This situation (assuming associativity) is discussed in [7]. Note that now an existentially closed algebra never contains an identity. Our Proposition 9 remains valid, and the same is true for Theorem 10 although the proof needs modifications. In $\theta(x, y, b, \vec{c}, \vec{d})$ we cannot use $\{x y=y x=1\}$ and replace it by $\left\{z=(y x)_{z}=z(y x) \mid z \in\left\langle x, y, b, c_{1}, \ldots, c_{n}\right\rangle_{K}\right\}$ which suffices for the 
argumentation.

Concerning our discussion of idempotents in associative $\Delta$-algebras, Theorem 14 can be strengthened by identifying $A$ with one of the components of the direct product $B$. Lemma 15 still holds and admits an easier proof. But Theorem 16 and Corollary 17 no longer make sense since conjugations are not defined in the absence of an identity.

\section{References}

[1] P.M. Cohn, "On the free product of associative rings", Math. 2. 71 (1959), 380-398.

[2] P.M. Cohn, Skew fiezd constructions (London Mathematical Society Lecture Note Series, 27. Cambridge University Press, Cambridge, London, New York, 1977).

[3] Warren Dicks, "The HNN construction for rings", J. Algebra 81 (1983), $434-487$.

[4] Ц.Е. Дидице [C.E. Dididze], "Неассоциативные свободные сумы алгебр с обьединенной поюалгєбой" [Nonassociative free sums of algebras with an amalgamated subalgebra], Soobšc. Akad. Nauk Gmizin. SSR 18 (1957), 11-17.

[5] Ц.Е. Дипидзе [C.Е. Dididze], "Неассоциатнвые свободые суммы алгебр с обьединенНой подалгеброй" [Nonassociative free sums of algebras with an amalgamated subalgebra], Mat. Sb. 43 (85) (1957), 379-396.

[6] Paul C. Eklof, "Ultraproducts for algebraists", Handbook of mathematical Zogic, 105-137 (Studies in Logic and the Foundations of Mathematics, 90. North-Holland, Amsterdam, New York, Oxford, 1977).

[7] Paul C. Eklof and Hans-Christian Mez, "ideals in existentially closed algebras", preprint.

[8] K.R. Goodearl, von Neumann regular rings (Monographs and Studies in Mathematics, 4. Pitman, London, San Francisco, Melbourne, 1979). 
[9] Graham Higman, B.H. Neumann and Hanna Neumann, "Embedding theorems for groups", J. London Math. Soc. 24 (1949), 247-254.

[10] Joram Hirschfeld and William H. Wheeler, Forcing, arithmetic, and division rings (Lecture Notes in Mathematics, 454. SpringerVerlag, Berlin, Heidelberg, New York, 1975).

[11] Nathan Jacobson, Structure of rings, revised edition (Colloquium Publications, 37. American Mathematical Society, Providence, Rhode Island, 1968).

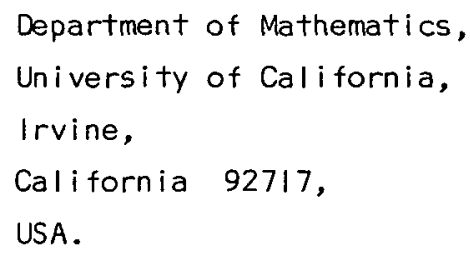

